\title{
Economic considerations of antifungal prophylaxis in patients undergoing surgical procedures
}

This article was published in the following Dove Press journal:

Therapeutics and Clinical Risk Management

I3 January 201 I

Number of times this article has been viewed

\section{Maria Adriana Cataldo \\ Nicola Petrosillo \\ Second Infectious Diseases Division, National Institute for Infectious Diseases, “Lazzaro Spallanzani”, Rome, Italy}

Correspondence: Maria Adriana Cataldo Second Infectious Diseases Division, National Institute for Infectious Diseases, "Lazzaro Spallanzani”, Via Portuense, 29200149 Rome, Italy Tel +39065 5170432

Fax +390655170486

Email adriana.cataldo@inmi.it

\begin{abstract}
Fungi are a frequent cause of nosocomial infections, with an incidence that has increased significantly in recent years, especially among critically ill patients who require intensive care unit (ICU) admission. Among ICU patients, postsurgical patients have a higher risk of Candida infections in the bloodstream. In consideration of the high incidence of fungal infections in these patients, their strong impact on mortality rate, and of the difficulties in Candida diagnosis, some experts suggest the use of antifungal prophylaxis in critically ill surgical patients. A clinical benefit from this strategy has been demonstrated, but the economic impact of the use of antifungal prophylaxis in surgical patients has not been systematically evaluated, and its cost-benefit ratio has not been defined. Whereas the costs associated with treating fungal infections are very high, the cost of antifungal drugs varies from affordable (ie, the older azoles) to expensive (ie, echinocandins, polyenes, and the newer azoles). Adverse drug-related effects and the possibly increased incidence of fluconazole resistance and of isolates other than Candida albicans must also be taken into account. From the published studies of antifungal prophylaxis in surgical patients, a likely economic benefit of this strategy could be inferred, but its usefulness and cost-benefits should be evaluated in light of local data, because the available evidence does not permit general recommendations.
\end{abstract}

Keywords: antifungal prophylaxis, cost-effectiveness, economics, surgery, fungal infection

\section{Introduction}

Fungi are a frequent cause of nosocomial infections, with an incidence that has significantly increased in recent years. ${ }^{1}$ It has been reported that Candida is responsible for $9 \%$ of all nosocomial bloodstream infections in the US ${ }^{2}$ and that it is the most common cause of invasive mycoses. ${ }^{3}$ Aspergillus species and other emerging fungi, such as Fusarium and Rhizopus species, are less frequently isolated, but may cause infections in immunocompromised surgical patients. Regarding Candida infections, C. albicans accounts for most nosocomial infections, although in some units up to $50 \%$ of Candida infections are due to species other than C. albicans. ${ }^{4,5}$

In the past, fungi were considered to be etiologic agents of infections essentially occurring in neutropenic patients, such as transplant recipients or those with cancer. More recently, a role for fungi has been recognized also in infections affecting critically ill patients, especially those who require intensive care unit (ICU) admission. Among ICU patients, surgical ones have a higher risk of Candida bloodstream infections. ${ }^{6}$ It has been reported that about two-thirds of all episodes of candidemia occur in ICUs and in surgical wards, ${ }^{1}$ and that Candida is the third most common isolate from blood in these wards. ${ }^{2}$ According to data from the National Epidemiology of Mycosis Survey, 
the overall rate of Candida bloodstream infections was 0.98 per 1000 surgical ICU patient-days and 1.42 per 1000 surgical ICU patient-days when a central venous catheter is in place. ${ }^{7}$ The higher incidence of fungal infections among surgical patients is due to the severity of their underlying diseases, impaired gastrointestinal mucosal integrity, frequency of treatment with broad-spectrum antibiotics, and parenteral nutrition. $^{3,4}$

The crude mortality rate for Candida bloodstream infection ranges from $40 \%$ to $60 \%,{ }^{8-10}$ and the mortality rate attributable to candidemia has been estimated at $25 \%-38 \%$. $^{11,12}$ However, the impact of Candida infections on mortality is still controversial. In a matched case-control study carefully adjusted for age, severity of illness, and underlying disease, it was demonstrated that candidemia did not significantly affect mortality in the ICU. ${ }^{13}$ In contrast, the results of a systematic review of studies examining the mortality rate related to candidemia suggest that most of the mortality is attributable to the infection itself. ${ }^{14}$

Surgical patients who develop Candida bloodstream infections have a mortality rate significantly higher than patients in surgical ICUs without this infection. ${ }^{7}$ Moreover, invasive candidiasis is associated with prolonged hospital stays. ${ }^{8-10}$ In a large cohort of ICU patients, it was calculated that nosocomial Candida infections were associated with an increase of 17 days and 8 days in length of hospital stay and ICU stay, respectively. ${ }^{15}$

A major problem affecting the management of fungal infection is the difficulty in making an early diagnosis, given the poor sensitivity of diagnostic tests. On the other hand, several efficacious antifungal agents are now available with a good safety profile.

The high incidence of fungal infections in surgical patients, impact on mortality rate, and abovementioned diagnostic difficulties have led some experts to suggest the use of antifungal prophylaxis in these patients. However, there is a lack of clear evidence of benefit from this strategy on the incidence of fungal infections and on mortality rates.

Although fungal infections, due to their high associated morbidity and mortality, have a strong impact on hospital costs and on health economic balances, the cost-benefit ratio of fungal prophylaxis strategy is not well defined. This review summarizes the available evidence for the economic impact of antifungal prophylaxis among surgical patients, and briefly reports the relevant efficacy data in light of the potential cost-benefit ratio.

\section{Which surgical patients are at greater risk for fungal infection?}

In spite of the identification of several risk factors associated with fungal infection among surgical patients, it is not possible to define a particular group of high-risk patients.

The probability of infection differs according to the type of surgical procedure. Patients who undergo abdominal surgery are at highest risk. Patients who undergo neurosurgical or ear, nose, and throat procedures are at the lowest risk, followed by orthopedic and gynecologic patients. ${ }^{7}$

A multicenter study including 4276 surgical patients identified previous surgery, acute renal failure, and receipt of parenteral nutrition as factors independently associated with increased risk of Candida bloodstream infections. ${ }^{7}$ Other risk factors for fungal infections in surgical patients are the severity of the underlying condition, ${ }^{16}$ duration of stay in the ICU, receipt of antibacterial agents, and use of a central venous catheter.

Colonization with Candida is another important risk factor for subsequent invasive infection. The role of Candida colonization on the development of subsequent infection has been specifically investigated in surgical patients, revealing that the intensity and duration of colonization are determining factors. ${ }^{17}$ Several risk-predictive models for invasive candidiasis among critically ill patients have been published. However, they showed suboptimal performance and were not validated in independent ICU cohorts. ${ }^{18}$

\section{Is antifungal prophylaxis efficacious in preventing infections in surgical patients?}

The effectiveness of antifungal prophylaxis is still debated in several patient categories. A number of studies on this topic have been published, and five meta-analyses have been done to reassess the effectiveness and safety of this strategy in surgical patients. The characteristics of the patients included and the prophylactic strategies analyzed differed between the published meta-analyses, but there was some overlap of the studies included. Here we report the main findings regarding the efficacy of antifungal prophylaxis in surgical patients.

A meta-analysis by Vardakas et al included six randomized, placebo-controlled trials containing 43-292 patients assessing the role of azoles in antifungal prophylaxis among high-risk surgical ICU patients. ${ }^{19}$ High-risk patients were defined as those having three or more risk factors associated with fungal infection, and patients undergoing transplantation were also 
included if they were expected to stay in the ICU for more than five days following surgery. Antifungal agents used in the studies were fluconazole $100-400 \mathrm{mg}$ with or without a loading dose and administered orally or intravenously, itraconazole $5 \mathrm{mg} / \mathrm{kg}$ orally, and ketoconazole $200 \mathrm{mg}$ orally, and with a variable duration of prophylaxis. A pooled analysis showed that patients receiving azole prophylaxis developed significantly fewer fungal infections, including candidemia, invasive fungal infections outside the bloodstream, and noninvasive fungal infections compared with placebo recipients. Mortality was significantly lower neither in patients undergoing antifungal prophylaxis, nor in the subgroup analysis conducted including only surgical patients. ${ }^{19}$

The meta-analysis by Cruciani et al included nine randomized controlled trials with 43-292 patients comparing systemic antifungal agents with controls in nonneutropenic adult ICU surgical or trauma patients. ${ }^{20}$ Antifungal agents used in the included studies were fluconazole 100-400 mg with or without a loading dose, administered orally or intravenously and ketoconazole $200 \mathrm{mg}$ administered orally or parenterally compared with placebo or with no treatment/ nystatin/clotrimazole. The length of prophylaxis differed among the studies. This pooled analysis also showed that the probability of Candida infection in patients who received antifungal prophylaxis was significantly lower. Interestingly, the metaregression showed that neither the azole prescribed nor the Acute Physiology and Chronic Health Evaluation (APACHE) II score influenced treatment effects. Moreover, this meta-analysis demonstrated a $79 \%$ reduction in mortality attributable to Candida infection in azole-treated patients. Overall mortality was also significantly reduced. ${ }^{20}$

The meta-analysis by Playford et al included 12 randomized controlled trials including 38-292 patients and evaluated the effect of any antifungal regimen in nonneutropenic critically ill patients. ${ }^{21}$ This meta-analysis also included nonsurgical patients, but the majority of those included were postsurgical ICU patients. Eight trials compared fluconazole $100-400 \mathrm{mg}$ with or without a loading dose administered orally or intravenously, with no antifungal therapy, and four compared ketoconazole 200-400 mg administered orally with no antifungal treatment or a nonadsorbable agent. The duration of prophylaxis differed among the studies. The fluconazole and ketoconazole trials analyzed separately did not show a significant reduction of mortality risk. Fluconazole significantly reduced the incidence of proven invasive fungal infections. When the trials were analyzed together, fluconazole and ketoconazole were found to reduce total mortality and invasive infections. Subgroup analysis of postsurgical patients demonstrated a significant reduction of proven invasive infections and mortality in the fluconazole and ketoconazole prophylaxis groups. ${ }^{21}$

The meta-analysis by Shorr et al included four randomized trials with 43-260 patients comparing fluconazole with placebo in critically ill surgical patients. There was heterogeneity among the trials with respect to fluconazole dosing (100-400 mg/day, with or without a loading dose), route of administration (orally or intravenously or both), and duration of the prophylactic regimen. The authors of this metaanalysis reported that prophylaxis significantly reduced the incidence of fungal infections but was not associated with a lower mortality rate. The incidence of candidemia was not significantly reduced in the fluconazole group. ${ }^{22}$

The meta-analysis by Ho et al included seven randomized controlled trials of fluconazole prophylaxis involving immunocompetent critically ill or high-risk surgical patients, excluding studies that included liver transplanted or neutropenic cancer patients. ${ }^{23}$ The numbers of patients included in the trials ranged from 43 to 260 , doses of fluconazole ranged from 100 to $800 \mathrm{mg} /$ day, and the duration of the prophylactic regimen varied between the studies. Candidemia was significantly less frequent in the fluconazole group, but hospital mortality and total length of hospital stay did not differ between the two groups. ${ }^{23}$

In conclusion, these meta-analyses showed a clinical benefit of antifungal prophylaxis in patients at different degrees of risk. Only two pooled analyses showed significantly lower mortality rates. However, due to the inadequate sample size of the trials and the heterogeneity in type and dosage of antifungal agent, duration of prophylaxis, and patient characteristics, it is not possible to identify a clear indication for a prophylactic antifungal strategy from these studies.

\section{Costs of antifungal prophylaxis}

When considering the economic balance of antifungal prophylaxis, it is necessary to take into account several cost components. First, the economic consequences of fungal infection must be considered. A multicenter Spanish study assessed the economic impact of Candida colonization and Candida infection in critically ill patients admitted to an ICU. Candida colonization resulted in an additional $€ 8000$ and Candida infection in almost $€ 16,000$ in direct costs, respectively. ${ }^{24}$

A prospective cohort study of Candida infections conducted in the medical and surgical ICUs of a large US hospital 
reported an unadjusted median cost of $\$ 41,832$ for patients with Candida infections and of $\$ 19,252$ for those without Candida infections. ${ }^{15}$ A cost per ICU patient-day of $\$ 230$ was also calculated. ${ }^{15}$

A multiple linear regression model was used to determine the cost of an ICU admission attributable to Candida infection and to verify if clinical factors other than fungal infection have an impact on the cost of an ICU admission. Candida infections were associated with a median increase in the cost of $\$ 21,590$ per patient and, in particular, with significantly increased costs in the service categories of room and board $(\$ 16,549)$, pharmacy (\$5501), laboratory (\$8216), supplies (\$3289), and physical and occupational therapy (\$2677). The variable that most strongly affected cost was the length of stay in the ICU. ${ }^{15}$ The results of a random effects model revealed that the daily ICU cost did not change after patients became infected with Candida, confirming that the increase in ICU cost is probably due to the increased length of stay rather than increased daily ICU cost. ${ }^{15}$

Another US study specifically assessed the estimated costs of care associated with candidemia over an entire hospital stay. ${ }^{10}$ The increase in cost of care during hospitalization was $\$ 44,536$ and $\$ 34,123$ in patients receiving private insurance and Medicare, respectively. ${ }^{10}$ The total cost included expenditure for diagnostic procedures, antifungal therapy, hospital stay, adverse drug reactions, and switch therapy because of adverse drug reactions. ${ }^{10}$ The largest component of total cost was due to protracted hospital stays. The results of a regression analysis performed to control for factors that may influence length of hospitalization showed that candidemia had the most impact. ${ }^{10}$

The acquisition cost of antifungal agent is also an important component of prophylaxis expenditure. Table 1 summarizes the costs of commercial available antifungal agents. Economic cost widely varies depending on the drug, the dosage used, route of administration, and total duration of prophylactic treatment. For example, published trials have reported fluconazole dosages of 100-400 mg/day preceded by a loading dose of $800 \mathrm{mg}$ and administration by the parenteral or enteral route, with different costs. Costs of antifungal agents vary between countries and hospitals, and may differ if the drug is administered during hospitalization, at a long-term care facility, or in an outpatient setting. Fluconazole is the drug most frequently used in prophylactic regimens. It is inexpensive and its pricing is expected to be reduced following the expiration of its patented life. The guidelines from the Infectious Diseases Society of America recommend fluconazole $6 \mathrm{mg} / \mathrm{kg} /$ day as an antifungal agent for prophylaxis in high-risk ICU patients. ${ }^{25}$

The antifungal profile and pharmacokinetic parameters of the echinocandins (caspofungin, micafungin, anidulafungin) could make them good choices for antifungal prophylaxis. However, these agents are expensive and need to be administered intravenously. Similarly, the high cost and need for parenteral infusion limits the use of lipid formulations of amphotericin B.

The economic cost of the side effects associated with antifungal agents must also be considered. The costs

Table I Costs of antifungal agents

\begin{tabular}{|c|c|c|}
\hline Antifungal agent (usual dosage) & Product cost (US\$) & Estimated daily cost* \\
\hline Amphotericin B $0.5-1.0 \mathrm{mg} / \mathrm{kg} / \mathrm{IV} /$ day & $\$ 12 / 50 \mathrm{mg}$ vial & $\$ 12-24$ \\
\hline L-Amphotericin B I-5 mg/kg/IV/day & $\$ 188 / 50 \mathrm{mg}$ vial & $\$ 263-1316$ \\
\hline \multirow[t]{2}{*}{ Fluconazole $400 \mathrm{mg} /$ day orally or IV } & $\$ 8 / 200 \mathrm{mg}$ tablet & $\$ 16$ for oral administration \\
\hline & $\$ 88 / 400 \mathrm{mg}$ IV & $\$ 88$ for IV administration \\
\hline \multirow[t]{2}{*}{ Itraconazole $200 \mathrm{mg} /$ day orally or $200 \mathrm{mg} /$ day IV } & $\$ 10 / 100 \mathrm{mg}$ capsules & $\$ 20$ for oral administration \\
\hline & $\$ 158 / 200 \mathrm{mg}$ vial & $\$ 158$ for IV administration \\
\hline Ketoconazole $400 \mathrm{mg} /$ day orally & $\$ 5 / 200 \mathrm{mg}$ tab & $\$ 10$ \\
\hline Posaconazole $600-800 \mathrm{mg} /$ day orally & $\$ 576 / 105 \mathrm{~mL}$ susp $(40 \mathrm{mg} / \mathrm{mL})$ & $\$ 82-110$ \\
\hline Voriconazole $6 \mathrm{mg} / \mathrm{kg} / \mathrm{IV} \mathrm{q} / 2 \mathrm{~h}$ first day, then & $\$ 121 / 200 \mathrm{mg}$ vial & $\$ 508$ first day then $\$ 255$ for \\
\hline $3 \mathrm{mg} / \mathrm{kg} / \mathrm{lV} \mathrm{q} \mid 2 \mathrm{~h}$ or $400 \mathrm{mg}$ orally q $\mathrm{2} 2 \mathrm{~h}$ on day I & $\$ 39 / 200 \mathrm{mg}$ tab & IV administration \\
\hline followed by $200 \mathrm{mg}$ orally $\mathrm{q} / 2 \mathrm{~h}$ & & $\begin{array}{l}\$ 156 \text { first day then } \$ 78 \text { for oral } \\
\text { administration }\end{array}$ \\
\hline $\begin{array}{l}\text { Anidulafungin } 100-200 \mathrm{mg} / \mathrm{IV} \text { first day followed } \\
\text { by } 50-100 \mathrm{mg} / \mathrm{IV} / \text { day }\end{array}$ & $\$ 1 / 2 / 50 \mathrm{mg}$ vial & $\$ 224-448$ first day then $\$ 112-224$ \\
\hline Caspofungin $70 \mathrm{mg} / \mathrm{IV}$ first day followed & $\$ 395 / 70 \mathrm{mg}$ vial & $\$ 395$ \\
\hline by $50 \mathrm{mg} /$ day IV & $\$ 395 / 50 \mathrm{mg}$ vial & \\
\hline Micafungin $50-150 \mathrm{mg} /$ day IV & $\$ 112 / 50 \mathrm{mg}$ vial & $\$ 112-336$ \\
\hline
\end{tabular}

Notes: "For a $70 \mathrm{~kg}$ patient. All cost data were extracted from the 2007 Drug Topics Redbook, Thomson Healthcare, Inc.

Abbreviations: L, liposomal; IV, intravenous; q, quondam; h, hours; cps, capsule; tab, tablet; susp, suspension. 
associated with drug-related adverse events depend on their incidence and severity, and are calculated taking into account the costs of several components, including loss of life-years, loss of quality life-years, long-term morbidity of selected adverse events, and an extended hospital stay due to an adverse event.

Fluconazole is generally well tolerated, with a very low incidence of serious adverse effects, and the new azoles and echinocandins have low toxicity rates. On the other hand, administration of amphotericin B or liposomal amphotericin is frequently associated with nephrotoxicity.

Some of the aforementioned meta-analyses explored the incidence of toxicity due to antifungal prophylaxis. Comparing azoles with placebo, there was no significant difference in the incidence of side effects. ${ }^{19}$ The pooled analysis that included trials comparing fluconazole or ketoconazole with no antifungal therapy or with nonadsorbable agents showed no difference in the occurrence of severe adverse events. Shorr et al, reviewing the safety of fluconazole prophylaxis, reported that rates of laboratory abnormalities were similar across all the studies that presented safety data, and did not differ between the intervention and placebo arms. No deaths were attributed to fluconazole administration, and intervention was stopped in only five patients (two on fluconazole and three on placebo) because of toxicity concerns. ${ }^{22}$

Another important economic consequence is the potential impact of prophylaxis on the emergence of antifungal resistance and on the prevalence of different fungal species. This effect is linked to the "ecological cost" of antifungal prophylaxis. Ecological costs represent the costs paid by future patients given inappropriate treatment due to the higher resistance to frequently used antifungal agents and by individual patient costs, ie, the costs of development of resistance in the individual patient, assuming that antifungal use will change the flora in the individual.

Above all, azole use has been linked to an increased incidence of fluconazole-resistant Candida isolates and to a shift towards isolation of Candida species other than C. albicans. Exposure to fluconazole is an already known risk factor associated with fluconazole resistance, ${ }^{26}$ and several investigators have reported an increased frequency of isolation of $C$. glabrata subsequent to azole use. ${ }^{27-29}$ These phenomena would have a strong impact on mortality from Candida infections. It has been shown that Candida species other than C. albicans have a worse prognosis. ${ }^{30-32}$ Similarly, in critically ill patients, infection with Candida strains resistant to fluconazole doubles the risk of death. ${ }^{33}$ As a consequence, a higher mortality rate would be responsible for an increase in costs. Moreover, in a setting with a higher prevalence of fluconazole-resistant Candida and of isolates other than C. albicans, the antifungal options would include the newer azoles and echinocandins, with a further significant increase in costs.

Resistance to echinocandins and the newer azoles would also increase as a consequence of greater use, so the cost-benefit of antifungal prophylaxis would be even more unfavorable. The role of antifungal prophylaxis in the emergence of resistant Candida and strains other than C. albicans is still controversial. An observational study in a surgical ICU where fluconazole prophylaxis had been utilized for three years, revealed that, when invasive candidiasis occurs, the causative agent is likely to have reduced susceptibility to fluconazole, whether or not it was ICU-acquired. ${ }^{34}$ However, three meta-analyses of azole prophylaxis reported neither development of resistance among the fungal isolates nor a shift from C. albicans toward other Candida species. ${ }^{19,20,23}$

The economic impact of the use of antifungal prophylaxis in surgical patients has not been systematically evaluated, but some authors have tried to extrapolate economic data from their investigations on antifungal prophylaxis.

In a double-blind, placebo-controlled trial evaluating the prophylactic use of enteral fluconazole to prevent Candida infections in 260 critically ill surgical patients, it was calculated that the number needed to treat to prevent one fungal infection was 9.5. ${ }^{35}$ The authors chose the enteral rather than the intravenous route for fluconazole because it costs less and has good bioavailability. The cost for a $400 \mathrm{mg}$ dose was $\$ 90$, whereas the calculated cost of Candida infections was $\$ 230$ per ICU patient-day. ${ }^{15}$ It was concluded that, given the low cost of enteral fluconazole and the low number of patients needed to treat to prevent fungal infections, the use of antifungal prophylaxis in this population is likely to be cost-effective.

Similarly Ho et al, with a baseline risk of candidemia that in the placebo arm of their meta-analysis was $4.5 \%$, calculated that 25 patients would need to be treated to prevent one episode of candidemia. Assuming a cost of $\$ 490$ for a 2-week prophylactic course of fluconazole per patient, the cost to prevent one episode of candidemia would be $\$ 12,250$, which is markedly less expensive than the calculated cost of each episode of candidemia. ${ }^{23}$

A study that investigated a risk-based fluconazole prophylaxis program included 36 patients admitted to a medical ICU. ${ }^{36}$ It was reported that the total cost of fluconazole prophylaxis was $\$ 6000$, which is much less expensive than the $\$ 567,000$ of excess health care costs associated with 
nine episodes of Candida bloodstream infection that occurred in the same unit before the start of the prophylaxis program. ${ }^{36}$ However, these data do not refer to surgical patients, and the study was conducted in a small ICU and consequently analyzed very few episodes of candidiasis.

Some authors have suggested that a more efficacious and economically feasible strategy could be to utilize colonization screening to guide the choice of patients who would benefit from antifungal prophylaxis. Candida colonization has been recognized as an important risk factor for subsequent infection, and it has been reported that, in the absence of fungal colonization, infection is unlikely. ${ }^{16}$ However, its positive predictive value of infection ranges between $12 \%$ and $18 \%$. Moreover, the variability in design of the published studies and in the reporting of the extent and duration of colonization does not allow us to give an exact indication on how to use colonization data for this purpose. Interestingly, the corrected colonization index, ie, ratio of highly positive samples to the total number of samples cultured, developed by Pittet et al, ${ }^{17}$ was utilized in a before-after study to guide the decision about which patients should receive pre-emptive treatment with fluconazole. ${ }^{26}$ The incidence of proven surgical ICU-acquired candidiasis significantly decreased while the incidence of imported candidiasis did not change. ${ }^{26} \mathrm{~A}$ corrected colonization index cutoff of 0.4 was defined as a probable indication of unnecessary prophylaxis. This strategy would probably be less expensive than if prophylaxis was administered to all patients, but the cost of mycological screening performed on all admitted patients is not negligible. No specific economic data are available for this strategy.

\section{Discussion}

The costs of fungal infections are very high, and surgical patients seem to be at greater risk for developing serious and invasive Candida infections, with a high mortality rate. The azoles are affordable, but the incidence of fluconazole resistance with isolates other than $C$. albicans needs to be investigated further because the available evidence does not exclude a strong impact on these phenomena. Similarly, further studies with adequate sample sizes are needed to define better the adverse effect profile of azole prophylaxis, particularly for hepatotoxicity.

Obviously, the economic balance of a prophylaxis strategy does not result from a simple opposition of the cost associated with Candida infections against the cost for antifungal agents and the related side effects.

The economic advantage of antifungal prophylaxis is linked to the incidence of infection and to the impact of prophylaxis on mortality. The fungal infection rates reported by the published studies are variable and consequently the data on efficacy and cost are not generalizable. Also, the incidence of invasive infections in surgical ICUs varies widely between hospitals, and all the trials have the limitations of being heterogeneous and including small patient populations.

If we can identify patients at very high risk for infection and administer a prophylactic regimen only to a selected population, the economic balance would probably be favorable. Unfortunately, there is not a defined group of patients at greatest risk as yet, and the strategy of colonization screening has many gaps and needs further investigation.

Some experts have suggested that prophylaxis would be advantageous when the rate of candidemia is at least $10 \% .{ }^{37}$ However, Ho et al reported a likely cost-benefit of antifungal prophylaxis pooling studies with a baseline candidemia risk of $4.5 \%$.

Recently published guidelines for the management of candidiasis recommend the use of antifungal prophylaxis in patients at high risk $(>10 \%)$ of invasive candidiasis hospitalized in ICUs with very high rates compared with the "normal rates" of $1 \%-2 \%{ }^{25}$

Another essential determinant of the economic balance is the actual impact of antifungal prophylaxis on the mortality rate. Not all published trials found lower mortality in the treated groups, and the pooled analyses performed by different investigators yielded conflicting results. The meta-analysis by Cruciani et al reported a reduction in mortality attributable to Candida infection and in overall mortality in the azole-treated group. ${ }^{20}$ In the meta-analysis by Playford et al, the subgroup analysis of postsurgical patients demonstrated a significant mortality reduction in the fluconazole/ketoconazole prophylaxis groups. ${ }^{21}$ The other meta-analyses failed to show a benefit on mortality rate..$^{19,22,23}$ The inability to detect a survival advantage with antifungal prophylaxis might be due to small sample sizes and to the selection of populations at insufficiently high risk to show a benefit. Future studies are needed with adequate sample sizes to define better the highest risk surgical population, and to assess the impact of prophylaxis on the incidence of infection and mortality.

A likely economic benefit of this strategy does emerge from the published studies on antifungal prophylaxis in surgical patients, but a specific cost-benefit analysis is lacking and is urgently needed.

The usefulness and the cost-benefit of antifungal prophylaxis in surgical patients should be evaluated in each surgical 
unit in light of local data concerning the incidence of invasive Candida infections and the clinical and epidemiological characteristics of the patient population treated, because the available evidence does not permit a universal recommendation.

It is important to underscore that, beyond prophylaxis with antifungal agents, standard measures to prevent nosocomial infections should always be applied both for their efficacy and for their low cost. Because transmission of Candida could occur via the hands of health care workers, especially during the care of catheters, all hospitals need to improve their adherence to hand hygiene. Also, extremely important is adherence to current recommendations for placement and care of central venous catheters. Finally, the correct use of antibiotics is another important component of candidemia prevention that would lead to a decrease in economic and ecological costs.

\section{Disclosure}

The authors report no conflicts of interest in this work.

\section{References}

1. Marchetti O, Bille J, Fluckiger U, et al. Epidemiology of candidemia in Swiss tertiary care hospitals: Secular trends, 1991-2000. Clin Infect Dis. 2004;38(3):311-320.

2. Wisplinghoff H, Bischoff T, Tallent SM, Seifert H, Wenzel RP, Edmond MB. Nosocomial bloodstream infections in US hospitals: Analysis of 24,179 cases from a prospective nationwide surveillance study. Clin Infect Dis. 2004;39(3):309-317.

3. National Nosocomial Infections Surveillance System. National Nosocomial Infections Surveillance (NNIS) System Report, data summary from January 1992 through June 2003, issued August 2003. Am J Infect Control. 2003;31(8):481-498.

4. Kao AS, Brandt ME, Pruitt WR, et al. The epidemiology of candidemia in two United States cities: Results of a population-based active surveillance. Clin Infect Dis. 1999;29(5):1164-1170.

5. Abi-Said D, Anaissie E, Uzun O, Raad I, Pinzcowski H, Vartivarian S. The epidemiology of hematogenous candidiasis caused by different Candida species. Clin Infect Dis. 1997;24(6):1122-1128.

6. Vincent JL, Anaissie E, Bruining H, et al. Epidemiology, diagnosis and treatment of systemic Candida infection in surgical patients under intensive care. Intensive Care Med. 1998;24(3):206-216.

7. Blumberg HM, Jarvis WR, Soucie JM, et al. National Epidemiology of Mycoses Survey (NEMIS) Study Group. Risk factors for candidal bloodstream infections in surgical intensive care unit patients: The NEMIS prospective multicenter study. The National Epidemiology of Mycosis Survey. Clin Infect Dis. 2001;33(2):177-186.

8. Wey SB, Mori M, Pfaller MA, Woolson RF, Wenzel RP. Hospitalacquired candidemia. The attributable mortality and excess length of stay. Arch Intern Med. 1988;148(12):2642-2645.

9. Wenzel RP. Nosocomial candidemia: Risk factors and attributable mortality. Clin Infect Dis. 1995;20(6):1531-1534.

10. Rentz AM, Halpern MT, Bowden R. The impact of candidemia on length of hospital stay, outcome, and overall cost of illness. Clin Infect Dis. 1998;27(4):781-788.

11. Tortorano AM, Peman J, Bernhardt H, et al. ECMM Working Group on Candidaemia. Epidemiology of candidaemia in Europe: Results of 28-month European Confederation of Medical Mycology (ECMM) hospital-based surveillance study. Eur J Clin Microbiol Infect Dis. 2004;23(4):317-322.
12. Almirante B, Rodríguez D, Park BJ, et al. Barcelona Candidemia Project Study Group. Epidemiology and predictors of mortality in cases of Candida bloodstream infection: Results from population-based surveillance, Barcelona, Spain, from 2002 to 2003. J Clin Microbiol. 2005;43(4):1829-1835.

13. Blot SI, Vandewoude KH. Estimating attributable mortality of candidemia: Clinical judgement vs matched cohort studies. Eur J Clin Microbiol Infect Dis. 2003;22(2):132-133.

14. Falagas ME, Apostolou KE, Pappas VD. Attributable mortality of candidemia: A systematic review of matched cohort and case-control studies. Eur J Clin Microbiol Infect Dis. 2006;25(7):419-425.

15. Pelz RK, Lipsett PA, Swoboda SM, et al. Candida infections: Outcome and attributable ICU costs in critically ill patients. J Intensive Care Med. 2000;15(5):255-261.

16. Pelz RK, Lipsett PA, Swoboda SM, Diener-West M, Hammond JM, Hendrix CW. The diagnostic value of fungal surveillance cultures in critically ill patients. Surg Infect (Larchmt). 2000;1(4):273-281.

17. Pittet D, Monod M, Suter PM, Frenk E, Auckenthaler R. Candida colonization and subsequent infections in critically ill surgical patients. Ann Surg. 1994;220(6):751-758.

18. Eggimann P, Ostrosky-Zeichner L. Early antifungal intervention strategies in ICU patients. Curr Opin Crit Care. 2010;16(5):465-469.

19. Vardakas KZ, Samonis G, Michalopoulos A, Soteriades ES, Falagas ME. Antifungal prophylaxis with azoles in high-risk, surgical intensive care unit patients: A meta-analysis of randomized, placebo-controlled trials. Crit Care Med. 2006;34(4):1216-1224.

20. Cruciani M, de Lalla F, Mengoli C. Prophylaxis of Candida infections in adult trauma and surgical intensive care patients: A systematic review and meta-analysis. Intensive Care Med. 2005;31(11):1479-1487.

21. Playford EG, Webster AC, Sorrell TC, Craig JC. Antifungal agents for preventing fungal infections in non-neutropenic critically ill and surgical patients: systematic review and meta-analysis of randomized clinical trials. J Antimicrob Chemother. 2006;57(4):628-638.

22. Shorr AF, Chung K, Jackson WL, Waterman PE, Kollef MH. Fluconazole prophylaxis in critically ill surgical patients: A meta-analysis. Crit Care Med. 2005;33(9):1928-1935.

23. Ho KM, Lipman J, Dobb GJ, Webb SA. The use of prophylactic fluconazole in immunocompetent high-risk surgical patients: A meta-analysis. Crit Care. 2005;9(6):R710-R717.

24. Olaechea PM, Palomar M, León-Gil C, et al. EPCAN Study Group. Economic impact of Candida colonization and Candida infection in the critically ill patient. Eur J Clin Microbiol Infect Dis. 2004;23(4): 323-330.

25. Pappas PG, Kauffman CA, Andes D, et al. Infectious Diseases Society of America. Clinical practice guidelines for the management of candidiasis: 2009 update by the Infectious Diseases Society of America. Clin Infect Dis. 2009;48(5):503-535.

26. Piarroux R, Grenouillet F, Balvay P, et al. Assessment of preemptive treatment to prevent severe candidiasis in critically ill surgical patients. Crit Care Med. 2004;32(12):2443-2449.

27. Berrouane YF, Herwaldt LA, Pfaller MA. Trends in antifungal use and epidemiology of nosocomial yeast infections in a university hospital. J Clin Microbiol. 1999;37(3):531-537.

28. Nguyen MH, Peacock JE Jr, Morris AJ, et al. The changing face of candidemia: Emergence of non-Candida albicans species and antifungal resistance. Am J Med. 1996;100(6):617-623.

29. Gleason TG, May AK, Caparelli D, Farr BM, Sawyer RG. Emerging evidence of selection of fluconazole-tolerant fungi in surgical intensive care units. Arch Surg. 1997;132(11):1197-1202.

30. Klingspor L, Törnqvist E, Johansson A, Petrini B, Forsum U, Hedin G. A prospective epidemiological survey of candidaemia in Sweden. Scand J Infect Dis. 2004;36(1):52-55.

31. Morgan J. Global trends in candidemia: Review of reports from 1995-2005. Curr Infect Dis Rep. 2005;7(6):429-439.

32. Dimopoulos G, Ntziora F, Rachiotis G, Armaganidis A, Falagas ME. Candida albicans versus non-albicans intensive care unit-acquired bloodstream infections: Differences in risk factors and outcome. Anesth Analg. 2008;106(2):523-529. 
33. Kovacicova G, Krupova Y, Lovaszova M, et al. Antifungal susceptibility of 262 bloodstream yeast isolates from a mixed cancer and noncancer patient population: Is there a correlation between in-vitro resistance to fluconazole and the outcome of fungemia? J Infect Chemother. 2000; 6(4):216-221.

34. Magill SS, Swoboda SM, Shields CE, et al. The epidemiology of Candida colonization and invasive candidiasis in a surgical intensive care unit where fluconazole prophylaxis is utilized: Follow-up to a randomized clinical trial. Ann Surg. 2009;249(4):657-665.
35. Pelz RK, Hendrix CW, Swoboda SM, et al. Double-blind placebo-controlled trial of fluconazole to prevent candidal infections in critically ill surgical patients. Ann Surg. 2001;233(4):542-548.

36. Faiz S, Neale B, Rios E, et al. Risk-based fluconazole prophylaxis of Candida bloodstream infection in a medical intensive care unit. Eur J Clin Microbiol Infect Dis. 2009;28(6):689-692.

37. Eggimann P, Garbino J, Pittet D. Epidemiology of Candida species infections in critically ill non-immunosuppressed patients. Lancet Infect Dis. 2003; 3(11):685-702.

\section{Publish your work in this journal}

Therapeutics and Clinical Risk Management is an international, peerreviewed journal of clinical therapeutics and risk management, focusing on concise rapid reporting of clinical studies in all therapeutic areas, outcomes, safety, and programs for the effective, safe, and sustained use of medicines. This journal is indexed on PubMed Central, CAS,
EMBase, Scopus and the Elsevier Bibliographic databases. The manuscript management system is completely online and includes a very quick and fair peer-review system, which is all easy to use. Visit http://www.dovepress.com/testimonials.php to read real quotes from published authors.

Submit your manuscript here: http://www.dovepress.com/therapeutics-and-clinical-risk-management-journal 